\title{
Aberrantly hypermethylated Homeobox A2 derepresses metalloproteinase-9 through TBP and promotes invasion in Nasopharyngeal carcinoma
}

\author{
Hsin-Pai Li, ${ }^{1,3, *}$, Chen-Ching Peng ${ }^{1, *}$, I-Che Chung ${ }^{2}$, Mei-Yuan Huang ${ }^{2}$, Shao-Tung \\ Huang ${ }^{1}$, Chia-Chun Chen ${ }^{2}$, Kai-Ping Chang ${ }^{4}$, Cheng-Lung Hsu ${ }^{5}$, Yu-Sun Chang ${ }^{1,2}$ \\ ${ }^{1}$ Graduate Institute of Biomedical Sciences, Chang Gung University, Taoyuan, Taiwan, Republic of China (ROC) \\ ${ }^{2}$ Molecular Medicine Research Center, Chang Gung University, Taoyuan, Taiwan, Republic of China (ROC) \\ ${ }^{3}$ Department of Microbiology and Immunology Medical School, Chang Gung University, Taoyuan, Taiwan, Republic of China \\ (ROC) \\ ${ }^{4}$ Department of Otolaryngology-Head and Neck Surgery, Chang Gung Memorial Hospital, Lin-Kou, Taiwan, ROC \\ ${ }^{5}$ Division of Hematology-Oncology, Chang Gung Memorial Hospital, Lin-Kou, Taiwan, ROC \\ * These authors contributed equally to this work.
}

Correspondence to: Hsin-Pai Li, email: paili@mail.cgu.edu.tw

Keywords: HOXA2, DNA methylation, NPC, MMP9

Received: September 4, $2013 \quad$ Accepted: November 2, $2013 \quad$ Published: November 4, 2013

This is an open-access article distributed under the terms of the Creative Commons Attribution License, which permits unrestricted use, distribution, and reproduction in any medium, provided the original author and source are credited.

\section{ABSTRACT:}

Nasopharyngeal carcinoma (NPC) is notorious for its high invasiveness and metastatic ability. In this study, we identified a differential hypermethylated transcription repressor, Homeobox A2 (HOXA2), which may render NPC cells invasive and metastatic. Aberrant hypermethylation of HOXA2 led to low RNA expression in NPC tumors and cells. Addition of methylation inhibitor 5'Aza restored HOXA2 RNA expression in NPC cells. Methylated HOXA2 promoter reduces the binding affinity of the transcriptional co-activator p300, causing transcriptional repression of HOXA2. In NPC cells, re-expression of ectopic HOXA2 was correlated with decreased invasive ability and reduced metalloproteinase MMP-9 RNA and protein expression. Promoter, ChIP and DNA-pull down assays indicated that HOXA2 competes with the transcription activator, TATA-box binding protein (TBP) for a recognition sequence near the MMP9 transcription start site, and suppresses MMP-9 transcription. Thus, HOXA2 acts as a suppressor or TBP-antagonist to inhibit MMP-9 expression; while methylationmediated inactivation of HOXA2 in NPC derepresses MMP-9 production and increases invasion of NPC cells. In NPC plasma samples, increased plasma EBV copy number was correlated with increased in cell-free HOXA2 hypermethylation and elevated MMP-9 levels. Plasma EBV DNA and methylated cell-free HOXA2 can be used as biomarkers for monitoring NPC treatment.

\section{INTRODUCTION}

Nasopharyngeal carcinoma (NPC) is a common head and neck cancer in Southeast Asia [1]. Diet, genetic background and Epstein-Barr virus (EBV) type II latent infection are strongly associated with NPC [2, 3]. A viral oncoprotein expressed during latent EBV infection, latent membrane protein 1 (LMP1), is considered to be a key factor in NPC development [4]. Previously, we demonstrated that LMP1 activates DNA methyltransferase
1 (DNMT1) and causes aberrant cellular DNA hypermethylation, leading to inactivation of the adhesion molecule, E-cadherin $[5,6]$. This indicates that the viral protein can silence gene via hypermethylation and promote NPC tumor progression. DNA methylation is essential for normal development, gene imprinting and $\mathrm{X}$ chromosome inactivation [7, 8]. However, aberrant DNA methylation has been associated with tumor suppressor gene (TSG) inactivation and tumorigenesis in many cancers [9]. We characterized differentially hypermethylated genes in NPC 
tumors and identified that the Homeobox A2 (HOXA2) gene is hypermethylated and down-regulated at the RNA level. There are $39 \mathrm{HOX}$ genes comprising four different chromosomal clusters A D in human. The HOX proteins are highly conserved transcription factors that share a similar DNA-binding domain called the homeodomain [10]. The $H O X$ genes are conserved among species and are important for body segment patterning [11, 12]. HOXA2 protein may suppress gene expression through the HOXA2-response element [13, 14]. The biological significance of $\mathrm{HOXA2}$ hypermethylation in NPC cells will be discussed.

\section{RESULTS}

\section{Differentially methylated HOXA2 correlates with low mRNA expression in NPC biopsies and cell lines}

To identify genes that were differentially methylated in NPC, we isolated genomic DNA from 4 tumor and non-tumor (T-N) paired NPC biopsies and analyzed the samples on TranSignal Methylation Promoter Arrays
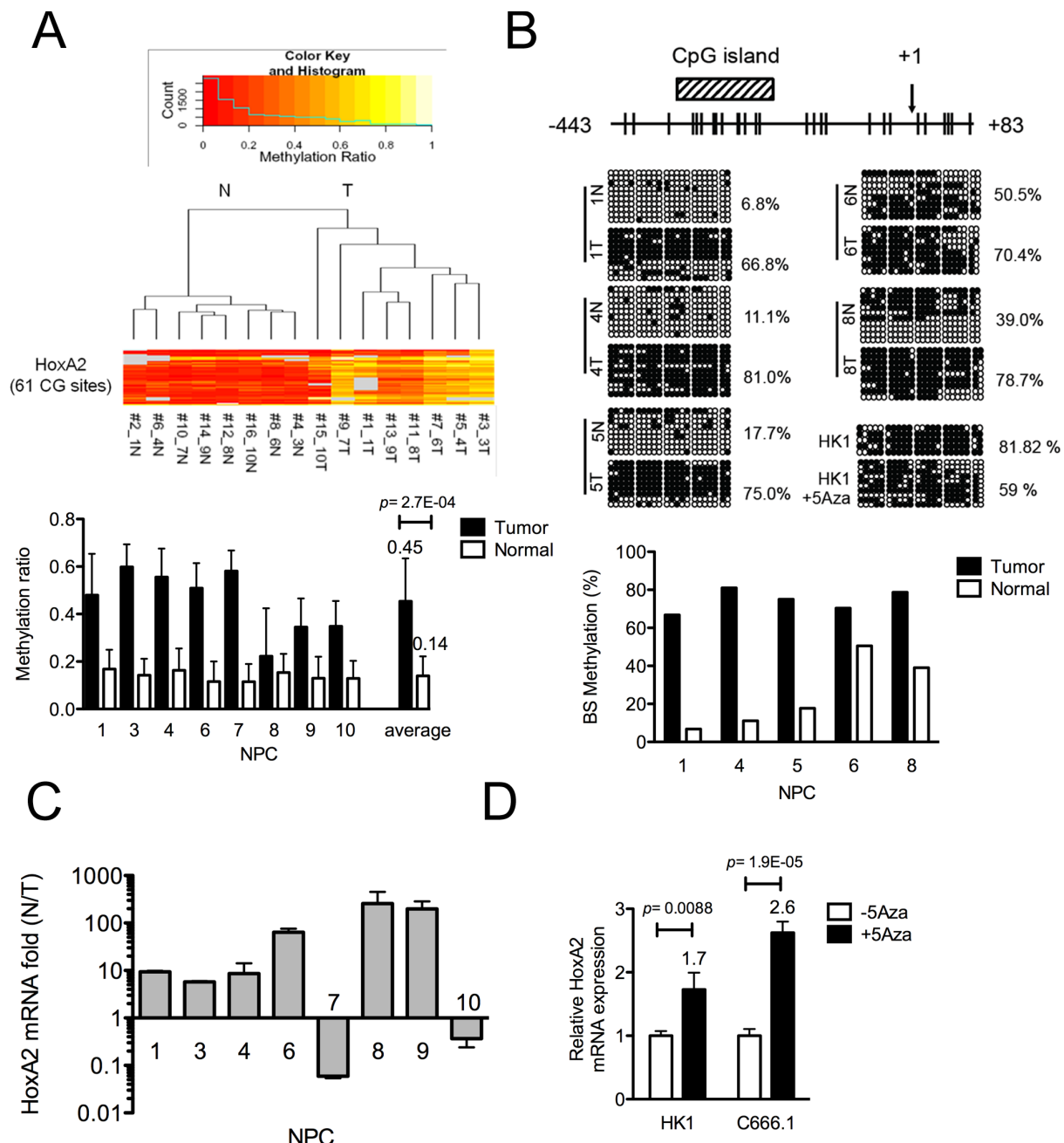

Figure 1: Identification of the HOXA2 gene as differentially methylated in NPC. (A) Three fragments covering the $61 \mathrm{CpG}$ sites in the HOXA2 promoter $(-1492 \sim+17)$ were amplified from 8 paired NPC samples and analyzed by MassArray $\left(\right.$ Sequenom $\left.{ }^{\circledR}\right)$. The colored histogram represents the $\mathrm{CpG}$ methylation ratio, ranging from $0(0 \%$ methylated $\mathrm{CpG}$ site, red) to 1.0 (100\% methylated $\mathrm{CpG}$ site, yellow). Each horizontal lane denotes the methylation ratio of a given CpG site in NPC tumor and adjacent normal tissues. The right panel shows the averaged methylation ratio of each sample. (B) Schematic map containing a CpG island (-345 -205, diagonal region) of the HOXA2 promoter, as adapted from the MethPrimer website [15]. The transcription start site $(+1)$ and $22 \mathrm{CpG}$ sites (vertical bars) are indicated. Bisulfite sequencing analysis was performed on $-443 \sim+83$ in 5 paired NPC clinical samples. Each horizontal row represents a single clone; the methylation percentages of at least 8 individual clones are indicated, with unmethylated (०) and methylated $(\bullet) \mathrm{CpG}$ sites. The lower panel shows the methylation percentage for each sample. (C) Q-RT-PCR analysis of HOXA2 mRNA expression was performed on the 8-paired NPC samples, and the results were normalized with respect to GAPDH expression. The columns represent the relative HOXA2 mRNA expression fold-change in log value (N/T). (D) Columns represent the Q-RT-PCR data of relative fold-change of restored HOXA2 mRNA expression normalized with respect to GAPDH expression in NPC cell lines (HK1, C666.1) (+/-) 10 $\mu \mathrm{M} 5$ ' Aza treatment. 
(Panomics). Among the tested 82 promoters, HOXA2 was found to be hypermethylated in NPC compared with normal adjacent tissues. In three of the four cases, the HOXA2 promoter hybridization signal in NPC tumors was stronger than that in the non-tumor counterparts (Fig. S1; left panel), indicating the amount of methylated $\mathrm{HOXA2}$ DNA was comparatively higher in tumors. In contrast, the promoter hybridization signal for the IRF7 gene (as negative control) was similar between tumor and nontumor samples (Fig. S1; right panel).

To confirm that the HOXA2 promoter is hypermethylated in NPC, we used MassARRAY mass spectrometry (Sequenom) to quantify the HOXA2 methylation status in $8 \mathrm{~T}-\mathrm{N}$ paired NPC biopsies (Supplementary MassARRAY methylation ratio). The MassARRAY methylation profile heat map for the 8 T-N paired NPC samples comprised $61 \mathrm{CpG}$ units $(-1421 \sim+17)$ (Fig. 1A; upper panel). NPC tumors with high methylation ratios or non-tumor tissues with low methylation ratios grouped separately in unsupervised clustering. The overall methylation ratio (the average of all tumors or nontumors) for the $8 \mathrm{NPC}$ tumors was 0.45 , whereas that of the adjacent normal counterparts was 0.14 (Fig. 1A; lower panel). Therefore, the overall fold-change of the average methylation ratios of the tumor and non-tumor samples was $3.3(* * *, \mathrm{p}<0.001)$, suggesting that the HOXA2 promoter is hypermethylated in NPC tumors.

To identify which $\mathrm{CpG}$ sites of the HOXA2 promoter are differentially methylated in NPC samples and cell lines, we performed bisulfite sequencing. MethPrimer web-based prediction [15] indicated that there was one $\mathrm{CpG}$ island (-345 -205) located near the transcription start site (TSS, +1) of HOXA2 promoter region (Fig. 1B). Our bisulfite sequencing results indicated that $-443 \sim+83$ of which contained the $\mathrm{CpG}$ island was hypermethylated in the NPC cells (85.8\% in HK1 and 94.8\% in C666.1). As shown in Fig. 1B, the methylation percentage of the same HOXA2 region was high in five clinical tumor samples (66.8 81\%, average 74\%) compared with their adjacent normal tissues $(6.8 \sim 50.5 \%$, average $25 \%)$.
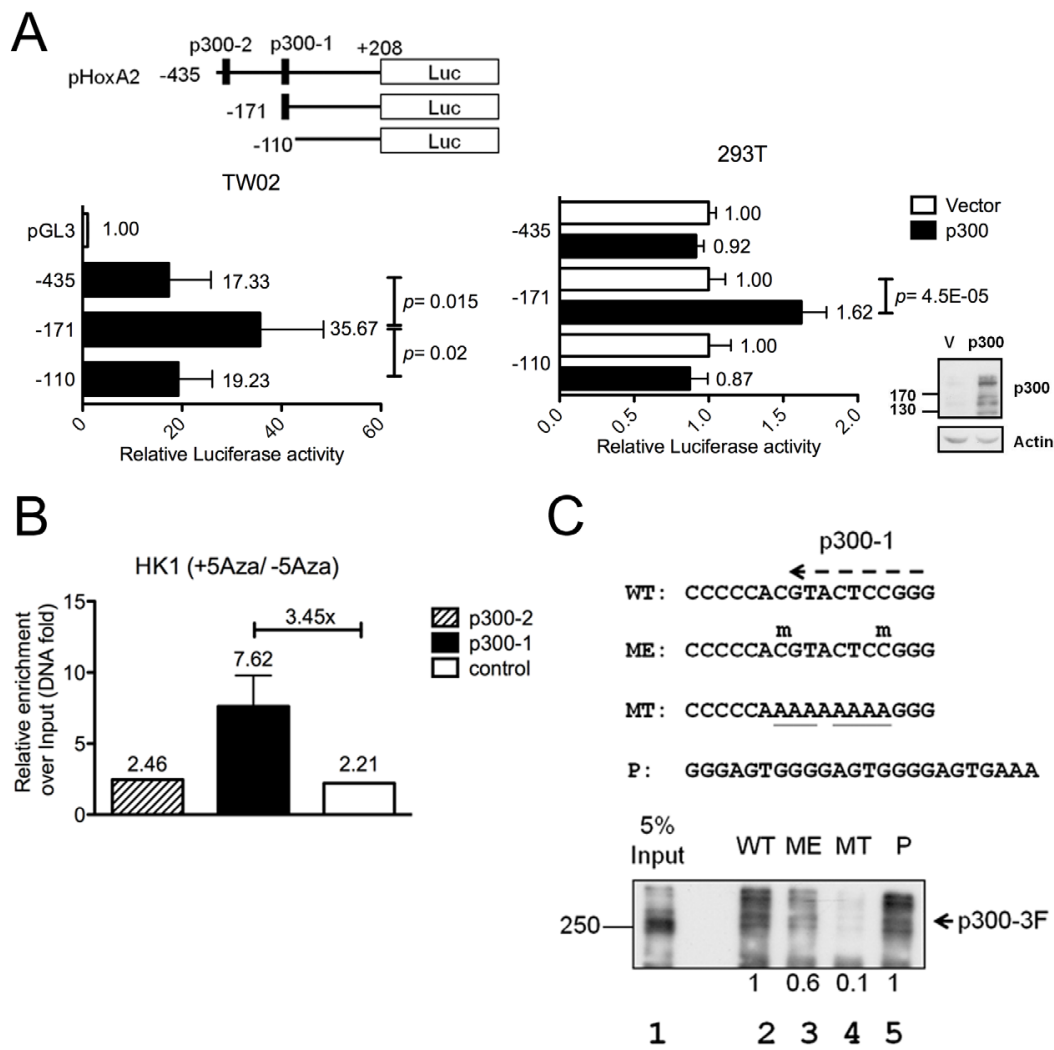

Figure 2: Methylation inhibits HOXA2 promoter activity by impairing binding of the transcription activator, p300. (A) Three HOXA2 promoter deletion clones and two putative $\mathrm{p} 300$ binding sites (p300-1 and $\mathrm{p} 300-2)$ are indicated. Different $H O X A 2$ promoter deletion clones were transfected into TW02 cells (left panel) or different HOXA2 promoter deletion clones and the pCMV/p300 expression clone were co-transfected into $293 \mathrm{~T}$ cells (right panel). Relative luciferase activities were detected $48 \mathrm{hr}$ post-transfection and all the luciferase reporter activities were normalized with respect to renilla activity. The expression levels of p300 and actin (internal control) were examined by Western blotting. (B) ChIP assays were performed with anti-p300 antibody or isotype IgG, using HK1 nuclear extracts treated with or without $10 \mu \mathrm{M}$ 5'Aza. Q-PCR was used to amplify p300-1 (-171 -55), p300-2 (-435 -254), and a downstream region lacking a p300 binding site $(-110 \sim+208)$. (C) DNA pull-down was used to analyze the binding affinity of exogenous Flag-tagged p300 (p300-3F) to wild-type (WT), methylated (ME), mutated (MT) HOXA2 p300-1 and a three copies of p300 consensus (P) biotinylated probes. Anti-Flag antibody was used to examine the amount of bound $\mathrm{p} 300-3 \mathrm{~F}$ in the immunoprecipitates and $5 \%$ inputs. Methylated cytosine is indicated by "m" above $\mathrm{C}$, and mutated sequences were underlined. 
Based on this differentially methylated region (DMR), we designed methylation-specific HOXA2 primers to quantify the methylation percentages of the same NPC clinical samples by quantitative methylation specific PCR (Q-MSP-PCR) analysis. As shown in Fig. $\mathrm{S} 2$, strong MSP-PCR products can be detected in all NPC tumors and a weak PCR product in one of the non-tumor samples, indicating again the methylation percentage in NPC tumors was higher than that of non-tumors. The quantitative values of Q-MSP methylation percentage of paired NPC tumor (T) and non-tumor (N) tissues were indicated in Fig. S3A, (T: 31.2 87.9\%, average 50.9\%; N: $1.8 \sim 28.1 \%$, average $6.7 \%$ ). Thus, the results from three different methylation-detecting assays all indicated that HOXA2 was differentially hypermethylated in NPC tumor tissues.

We then used quantitative (Q)-RT-PCR to evaluate the HOXA2 mRNA levels in the same NPC tumor tissues, and found that generally $H O X A 2$ mRNA levels were reduced 5- 210-fold (compared with the corresponding adjacent normal tissues) in six out of the eight NPC tumors (Fig. 1C). The relative quantitative values of
HOXA2 mRNA level of paired NPC tissues were indicated in Fig. S3B. Our data also indicated that the HOXA2 DNA methylation level (Q-MSP methylation \%) was negatively correlated with the relative RNA expression levels $[-\Delta \mathrm{Ct}=$ $\left.-\left(\mathrm{Ct}_{\text {HOXA2 }}-\mathrm{Ct}_{\text {GAPDH }}\right)\right]$ in NPC samples (Fig. S3C; correlation $\mathrm{r}=-0.45)$. Furthermore, the HOXA2 mRNA levels in the two NPC cells, C666.1 and HK1, could be restored by addition of the demethylation agent, 5'Aza (Fig. 1D), suggesting that DNA methylation was responsible for the transcriptional repression of $H O X A 2$ in NPC cells.

\section{DNA methylation suppresses HOXA2 promoter activity by impairing the binding of the transcription activator, $\mathbf{p 3 0 0}$.}

To determine whether DNA methylation per se can silence $H O X A 2$ promoter activity, we performed patch methylation assays [16]. The HOXA2 promoter was PCR-generated and in vitro methylated by the methylase, SssI. Methylation was verified by digestion with the methylation-sensitive restriction enzyme, BstUI (Fig. S4A). Methylated (pHOXA2 ${ }^{\mathrm{me}}$ ) and unmethylated

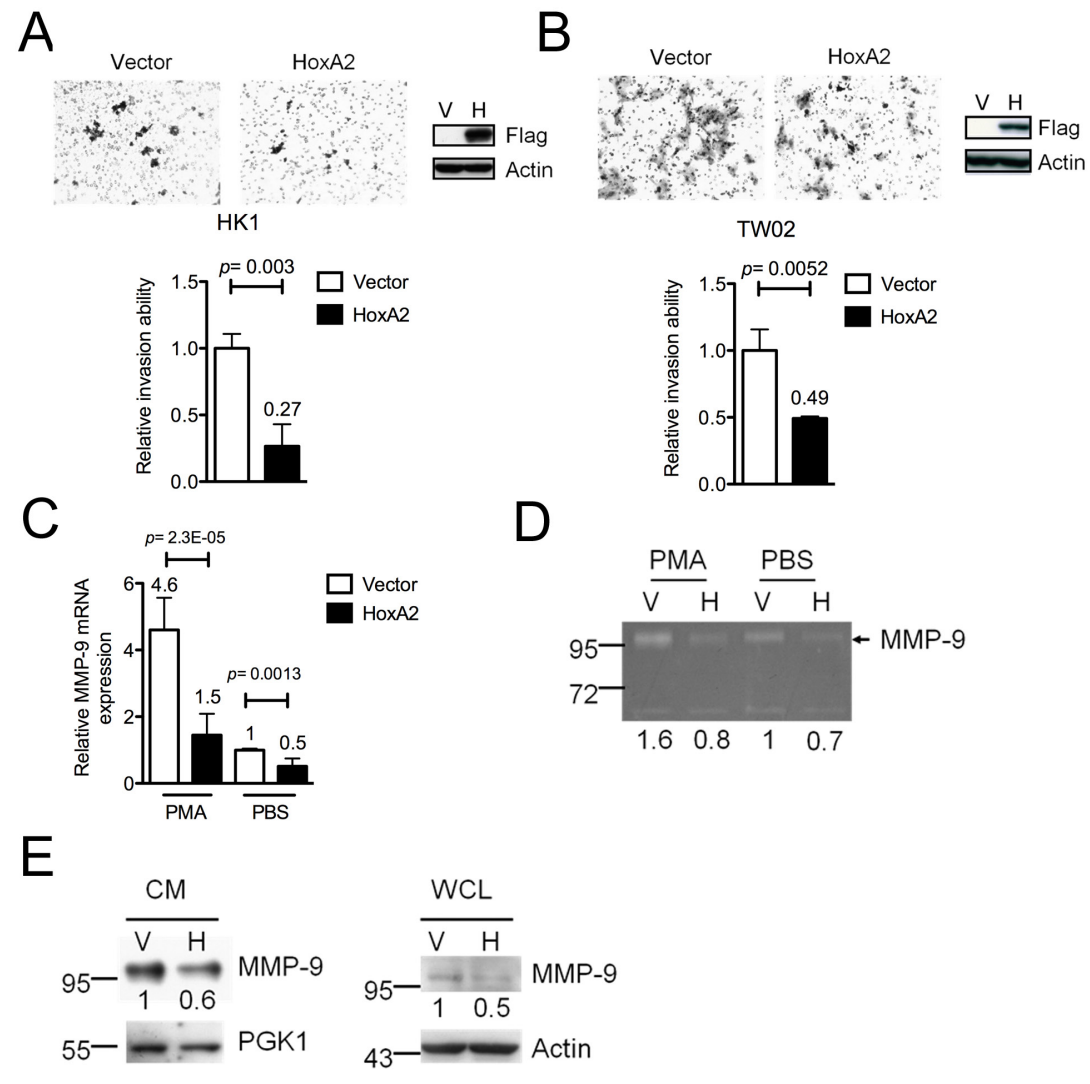

Figure. 3: HOXA2 inhibits cell invasion by down-regulating MMP-9 expression in NPC cells. The cell invasion abilities of (A) HK1 and (B) TW02 cells stably expressing HOXA2 and vector were analyzed. Migrated cells were counted from 10 different microscopic fields, and Western blot analysis was used to confirm the expression of exogenous HOXA2 (anti-Flag) and the internal control (anti-actin). (C) MMP-9 mRNA, (D) MMP-9 enzymatic avtivities and (E) MMP-9 protein in cultured medium (CM) and whole cell lysate (WCL) of vector control (V) and HOXA2-expressing $(\mathrm{H}) \mathrm{HK} 1$ cells were measured by QRT-PCR, gelatin zymography and Western blotting. Cells were either treated with or without PMA. PGK1 and actin were the protein loading control in CM and WCL, respectively. (A-E) Three independent experiments were performed in duplicate, and the results are shown as mean $\pm \mathrm{SD}(* \mathrm{p}<0.05, * * \mathrm{p}<0.01$, and $* * * \mathrm{p}<0.001)$. 


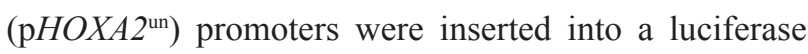
reporter and separately transfected into $293 \mathrm{~T}$ cells. The

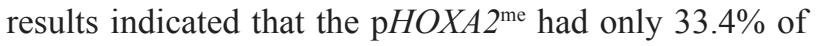

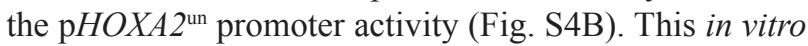
result supports our observation in clinical NPC samples that DNA methylation significantly suppresses $\mathrm{HOXA2}$ promoter activity.

To test whether methylated $\mathrm{CpG}$ sites impair the binding of transcription factors, we used TFSEARCH to analyze transcription-factor-binding motifs in the $\mathrm{HOXA2}$ promoter, focusing on the DMR identified by our bisulfite sequencing analysis. One of the selected transcription factors was p300, a transcriptional co-activator that functions as a histone acetyltransferase and regulates chromatin remodeling [17]. Two putative p300 binding sites close to the TSS were identified in the HOXA2 promoter: p300-1 (-137) and p300-2 (-290). To examine whether these $\mathrm{p} 300$ sites were crucial for $H O X A 2$ promoter activation, $\mathrm{pHOXA2-Luc} \mathrm{deletion} \mathrm{reporters} \mathrm{were} \mathrm{generated}$ (Fig. 2A, upper panel) and transfected into TW02 cells (have high-level endogenous p300 expression). Higher relative luciferase activity was detected from $\mathrm{pHOXA2(-171)} \mathrm{35.67-fold} \mathrm{versus} \mathrm{pHOXA2(-435)} \mathrm{17.33-}$ fold, and pHOXA2(-110) 19.23-fold (Fig. 2A, left panel), suggesting that $\mathrm{p} 300-1$ is important for $H O X A 2$ promoter activity. Similar results were obtained when a p300 expression plasmid was co-transfected into $293 \mathrm{~T}$ cells (have low-level endogenous p300) along with the same $\mathrm{p} H O X A 2-\mathrm{Luc}$ deletion reporters. The promoter activity

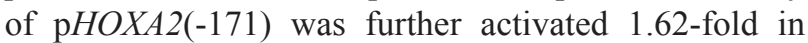
the presence of p300 (Fig. 2A, lower panel), while the activities of the two other promoters were unaltered in the presence of $\mathrm{p} 300$. Thus, our promoter assays indicate that p300 activates the $H O X A 2$ promoter mainly via the p3001 site.

To confirm whether the $\mathrm{p} 300$-binding affinity of the $H O X A$ promoter could be affected by DNA methylation, we performed quantitative ChIP assays using p300 antibody and HK1 cells with or without 5'Aza treatment. Our data showed that DNA demethylating agent, 5'Aza, enhanced the binding of p300 to the p300-1 site (by 3.45fold) but not the p300-2 site (1.1-fold) on the HOXA2 promoter when compared with the untreated control cells (Fig. 2B). These results indicate that $\mathrm{p} 300$ binds to the p300-1 site but not to the p300-2 site.

Next, we used DNA pull-down experiments to test
A
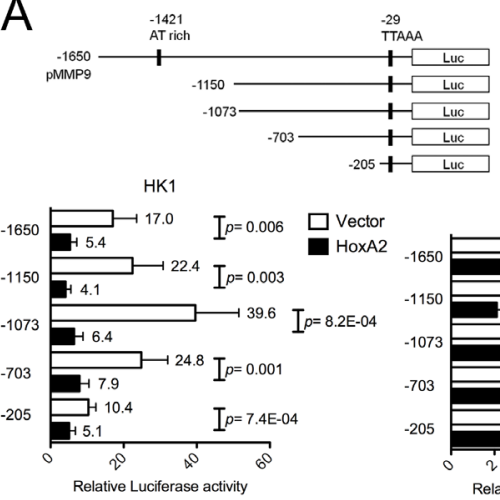

B WT: CCTTAAAGC MT: $C C$ GGGGGGC HK1 MMP-9 $(-205 \sim+150)$ $\stackrel{p=0.034}{\longmapsto}$

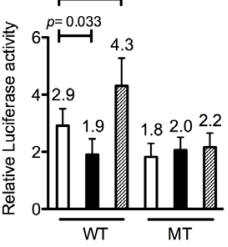

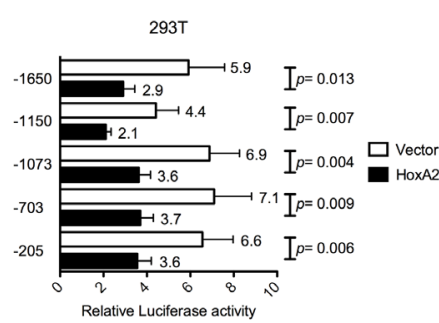

Relative Luciferase activity
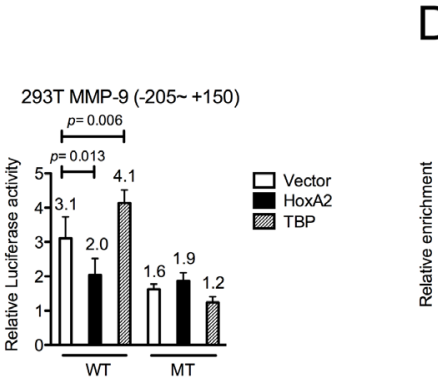

D

C
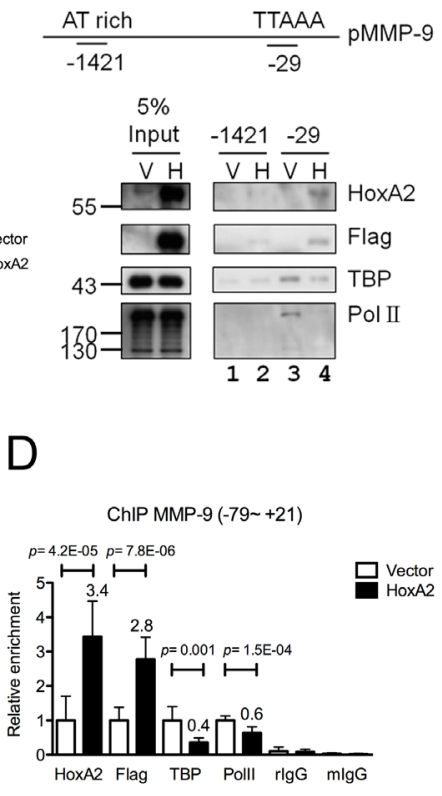

Figure 4: Direct binding of HOXA2 to the MMP-9 TATA box region interferes with the TBP-mediated activation of MMP-9. (A) MMP-9 promoter deletion clones and the pCMV/HOXA2 expression vector or (B) mutated promoter MT (-205 +150) and pCMV/HOXA2 or pCMV/TBP were co-transfected into HK1 and 293T cells, and luciferase reporter activity was detected. (C) In vitro binding of HOXA2, TBP and RNA PolII on the MMP-9 promoter was determined by DNA pull-down assays. Biotinylated probes containing the MMP-9 TATA box (-39 -15) or an upstream AT-rich region (-1421 -1397) were incubated with nuclear extracts from HK1 cells stably expressing vector or HOXA2. Western blotting was used to examine the expression of HOXA2, Flag-tagged HOXA2, TBP and PolII in the immunoprecipitates and 5\% inputs. (D) ChIP assays were performed with antibodies against HOXA2, Flag, TBP, PolII, or isotype IgGs using nuclear extracts from HK1-expressing or control cells. Q-PCR was used to amplify the MMP-9 promoter TATA box $(-39 \sim-15)$. (A-E) The presented data reflect three independent experiments, and the results are shown as mean $\pm \mathrm{SD}(* \mathrm{p}<0.05, * * \mathrm{p}<0.01$, and $* * * \mathrm{p}<0.001)$. 
whether DNA methylation of two CpG sites on $H O X A 2$ p300-1 site could affect the p300-binding affinity. We synthesized double-stranded biotinylated oligomers containing the p300-1 site with unmethylated (WT) or methylated (ME) CpG sites; mutated p300 sequence (MT) as negative control; and three copies of p300 consensus sequence $(\mathrm{P})$ as positive control. Biotinylated probes were bound to nuclear extracts overexpressing Flag-tagged p300 (p300-3F), precipitated by streptavidin beads followed by Western blotting. The results showed that p300-3F had a relative strong binding with the WT probe (Fig. 2C, lane 2, considered as 1) and the positive control (lane 5) but had a weak binding with the ME probe (lane 3, 0.6-fold). However, the binding of p300 was abolished towards the mutant MT probe (lane 4). These results indicate that p300 has a lower binding affinity for the methylated p300-1 binding site of the HOXA2 proximal promoter, resulting in lower $H O X A 2$ expression. Conversely, demethylation of the $H O X A 2$ promoter by 5'Aza appears to promote binding of p300 to the p300-1 site and restore HOXA2 expression.

\section{Functional analysis of HOXA2-expressing NPC cell lines}

Silencing of TSGs via promoter hypermethylation is an early event in various cancers [7]. If inactivation of $H O X A 2$ contributes to NPC formation, then re-expression of $H O X A 2$ in NPC cells might reverse the oncogenic features of NPC cells. A recombinant lentivirus expression system was used to stably overexpress HOXA2 or the vector control in HK1 and TW02 cells. Proliferation, colony formation and cell invasion assays were used to
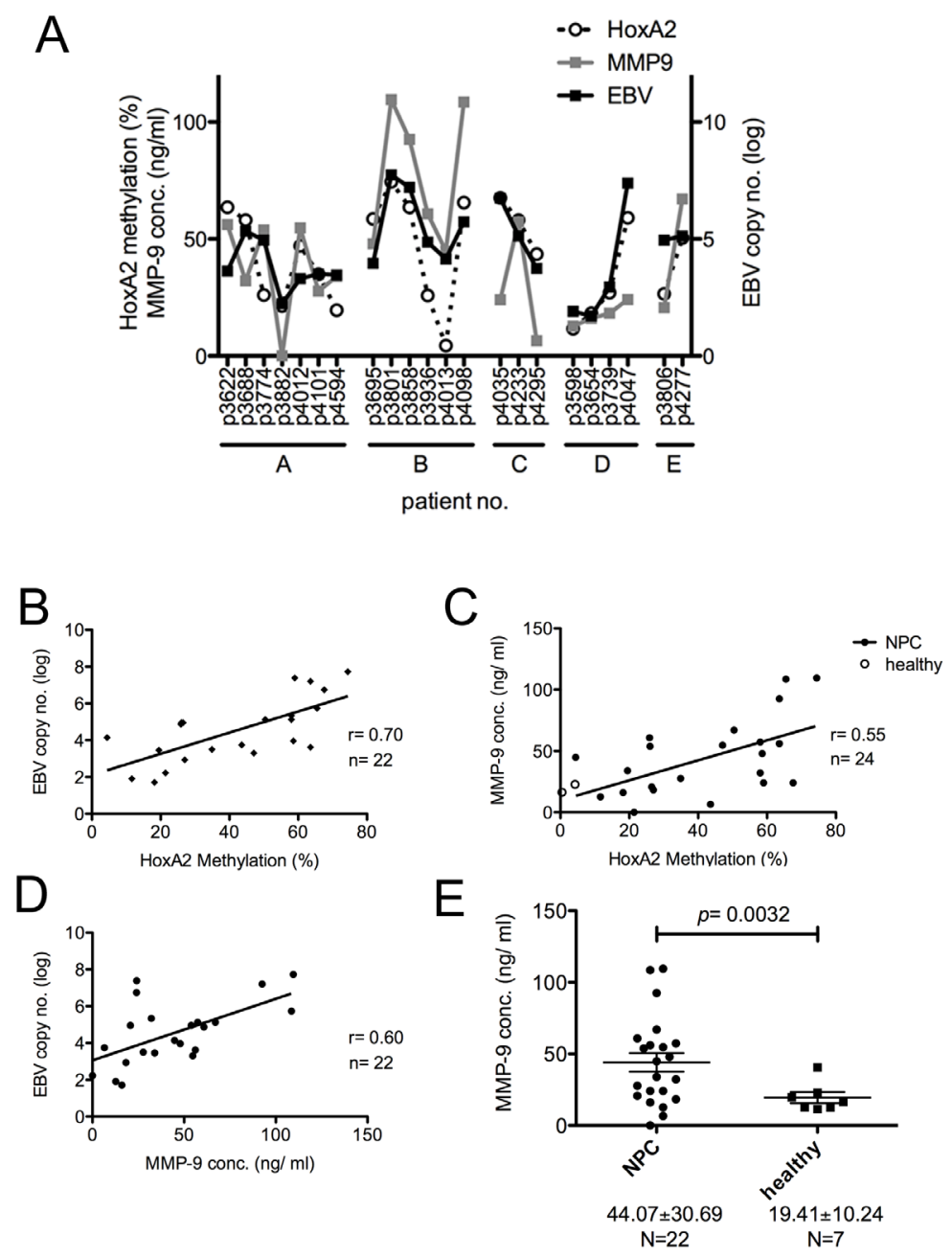

Figure 5: Correlation of EBV copy number, HOXA2 methylation status and MMP-9 concentration in plasma samples from NPC patients. Plasma was collected from five NPC patients at different time intervals (1 3 months) after standard NPC treatments (including radiation and chemotherapy) were administered according to the CGMH guidelines ( $\mathrm{N}=22)$. (A-D) Correlations were observed between the log EBV DNA copy number, HOXA2 methylation status and plasma MMP-9 concentration. Pearson's correlation coefficients (r) are indicated. (E) Plasma MMP-9 concentrations from 22 NPC samples and seven healthy donors were measured by ELISA. The results are shown as mean $\pm \mathrm{SD}(* * \mathrm{p}<0.01)$. 
examine the biological functions of HOXA2 in vitro. No significant difference was observed in the growth rate and colony formation of HOXA2-expressing HK1 (Fig. S5A) or TW02 cells (Fig. S5B) compared to control cells. However, cell invasion assays indicated that fewer HOXA2-expressing HK1 cells (0.27-fold) and TW02 cells (0.49-fold) invaded across the membrane compared with control cells (Fig. 3A) demonstrating that HOXA2 expression inhibits the cell invasion ability. And this inhibition was not due to a difference in cell proliferation between HOXA2-overexpressing and control cells.

\section{HOXA2 down-regulates MMP-9 expression and enzymatic activity}

Previous reports indicated that cell invasion ability can be promoted by overexpression of matrix metalloproteinases (MMPs), which degrade various extracellular components [18]. Next, we analyzed 22 $M M P s$ expression from the cDNA microarray (Affymetrix) data of nine NPC tumors versus one combined adjacent normal (control). We found that 8 out of 22 MMPs were overexpressed ( $>1.5$-fold) in NPC tumors when compared with the control (Table S3). The top of the MMPs list was MMP-9 (40-fold higher in NPC tumors compared with control), one of the best-studied proteinases involved in promoting tumor invasion and metastasis. In fact, MMP9 has been reported to facilitate NPC tumorigenesis in many studies [19-21]. As HOXA2 reportedly functions as a transcriptional repressor during development [13, 22], we hypothesized that HOXA2 might repress MMP-9 expression. We examined the mRNA, protein expression level and enzymatic activity of MMP-9 in HOXA2expressing and control NPC cells. As expected, MMP-9 mRNA levels were significantly reduced (to $\sim 50 \%$ ) in HOXA2-expressing HK1 cells compared with control cells (Fig. 3C). Even in the presence of phorbol myristate acetate (PMA), a known chemical that activates MMP-9 expression [23], the mRNA level of MMP-9 was again significantly lower in HOXA2-expressing HK1 cells (1.45-fold) compared with control cells (4.60-fold). Western blotting showed that in the presence of HOXA2 the protein level of MMP-9 was downregulated in both cultured medium and whole cell lysates (Fig. 3E). Gelatin zymography assays indicated that HOXA2 expression moderately suppressed the enzymatic ability of MMP-9 (0.73-fold) with respect to its activity in control cells (Fig. 3D, compare lane 3 and 4). In PMA-treated cells, HOXA2 overexpression was associated with a $\sim 50 \%$ reduction in MMP-9 enzymatic activity (0.82-fold) compared with control cells (1.56-fold) (Fig. 3D, compare lane 1 and 2). Collectively, HOXA2 represses $M M P-9$ RNA and protein expression in NPC cells.

\section{HOXA2 antagonizes TBP by binding to TATA- box and suppresses MMP-9 transcription}

To investigate whether HOXA2 is a transcriptional repressor of $M M P-9$, we generated various $M M P-9$ promoter deletion clones containing potential HOXA2binding sites. HOXA2 binds to the TAAT target sequence to down-regulate the transcription of its target genes $[14,24,25]$. A putative HOXA2-response element was identified at the MMP-9 promoter (-1421). We constructed

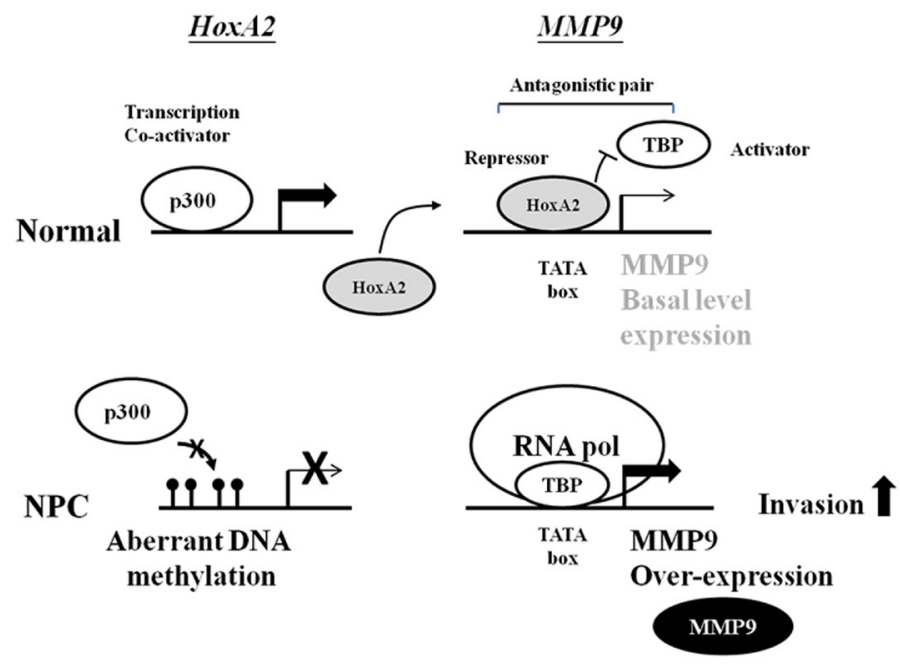

Figure 6: Schematic model of silencing of the transcription repressor HOXA2 by aberrant hypermethylation enhances invasion via MMP-9 activation in NPC. In normal cells, transcription of $H O X A 2$ is activated by p300 binding. Subsequently, HOXA2 binds to the MMP-9 TATA-box and interferes the binding of TBP resulting in suppression of $M M P-9$ expression. In NPC cells, methylation of the HOXA2 impairs the p300 binding thereby inactivates the HOXA2 gene. In the absence of transcription repressor HOXA2, TBP and RNA polII can bind to the TATA-box of $M M P-9$ and activate $M M P-9$ expression. Elevated MMP-9 level, in turn, promotes the invasiveness of NPC cells. 
five $M M P-9$ promoter Luc reporters (-1650, -1150 , $-1073,-703$ and $-205 \sim+150$; Fig. 4A). MMP-9 promoter clones and HOXA2-expressing or control vectors were co-transfected into HK1 and 293T cells. Luciferase assays revealed that the promoter activities of all five $M M P-9$ promoter deletion clones, including the shortest $-205 \sim+150$, were suppressed in HOXA2-overexpressing cells compared with vector control (Fig. 4A). Sequence analysis revealed that the only possible HOXA2 binding site within the $(-205 \sim+150) M M P-9$ promoter was the TATA-like sequence, TTAAA (-29), next to the TSS. Luciferase assays indicated that co-transfection of either HOXA2 or TBP expression clones with the shortest $M M P-9$ promoter reporter could either suppress slightly or activate the promoter activities (Fig. 4B). To test whether the TATA-like sequence was responsible for the transcriptional repression or activation, by HOXA2 or TATA-binding protein (TBP), respectively, we generated a site-directed MMP-9 promoter mutant, denoted as -205 MT, in which the TTAAA sequence was mutated to GGGGG. Luciferase assays demonstrated that this mutation abolished the HOXA2-mediated repression or TBP-mediated activation of MMP-9 transcription when compared to wild type reporter (Fig. 4B) suggesting that the TATA-like sequence is crucial for the recognition of both HOXA2 and TBP.

To further clarify the HOXA2-binding sites in the $M M P-9$ promoter, we performed DNA pull-down experiments using the two double-stranded biotinylated oligonucleotide probes containing the putative HOXA2response element (-1421) and the TATA-like sequence $(-29)$, respectively. Biotinylated probes were bound to nuclear extracts of vector- or HOXA2-expressing HK1 cells, purified by streptavidin beads followed by Western blotting. The results revealed that HOXA2 had a relatively strong affinity toward TATA-like sequence (-29) in HOXA2-expressing HK1 extracts (Fig. 4C; antiHOXA2, anti-Flag, lane 4) when compared with vector control extracts (Fig. 4C, lane 3). Little or no HOXA2 binding was observed to the putative HOXA2-response element (-1421) in vector- or HOXA2-expressing HK1 extracts (Fig. 4C, lanes 1 and 2) indicating that HOXA2 binds to the $M M P-9$ promoter at (-29) but not (-1421). Based on these findings, we hypothesized that HOXA2 may compete with TBP to block the binding of RNA polymerase II (RNA PolII). Additional Western blot analyses using the same DNA pull-down lysates showed that significant TBP and RNA PolII bound to the TATAlike sequence (-29) probes (Fig. 4C; anti-TBP and antiRNA PolII, lane 3 ) in the absence of HOXA2 expression, but both bound only weakly in the HOXA2-expressing HK1 extracts (Fig. 4C, lane 4). This suggests that the TBP and HOXA2 factors may compete for the same binding site at (-29). The presence of HOXA2 could interfere or compete with the binding of TBP and RNA PolII to the $M M P-9$ promoter.
To address whether HOXA2 and TBP can directly bind to the $M M P-9$ TATA-like sequence, we used purified recombinant His-tagged HOXA2 and TBP proteins to perform EMSAs. We found that recombinant HOXA2 and TBP proteins could both bind directly to the $M M P$ 9 TATA box biotin-labeled probe (Fig. S6, solid arrows, lane 2 and 5). Furthermore, the two proteins might form higher-molecular-weight dimers or multimers with the probes (Fig. S4, dashed arrows, lane 2 and 5). Incubation with the corresponding antibodies led to the formation of a supershifted complex and a marked decrease in the intensity of the lower band (Fig. S6, asterisks, lane 3 and 6). However, when the TTAAA core sequence was mutated, the binding affinity between the recombinant HOXA2 or TBP proteins and mutant probe dropped dramatically (Fig. S6, lane 11 and 12).

To test whether HOXA2 could interfere with TBP binding and inhibit MMP-9 transcription in vivo, we performed quantitative ChIP assays on the $M M P-9$ promoter in HOXA2-expressing and vector control cells. In HOXA2-expressing cells, HOXA2 bound to the MMP9 TATA box region and reduced the binding of TBP and RNA PolII to the same region (Fig. 4D), compared with the binding in vector control cells. These results indicate that HOXA2 may antagonize TBP binding and RNA PolII recruitment to the $M M P-9$ TATA box region, thereby decreasing $M M P-9$ transcription.

\section{HOXA2 methylation status positively correlates with EBV copy number and MMP-9 levels in plasma from NPC patients}

It is generally accepted that the cell-free DNA (cfDNA) content is higher in cancer patients compared with healthy people [26]. To test whether the levels of (i) EBV copy number, (ii) HOXA2 methylation ratio and (iii) MMP-9 levels are inter-correlated in a given sample, we obtained 3 5 follow-up plasma samples from five treated $\mathrm{NPC}$ patients (Fig. 5, designated $\mathrm{A} \sim \mathrm{E} ; \mathrm{N}=22$ ), and $\mathrm{EBV}$ copy number in the plasma was monitored by q-PCR; and cfDNA was extracted from the samples and methylation specific-high resolution melting (MS-HRM) (Fig. S7 and Table S4) and q-PCR was performed to detect the HOXA2 methylation ratio; and the MMP-9 levels of the plasma samples were detected by the ELISA. Moderate to strong positive correlations were observed when we compared the levels of any two of the three factors (Fig. 5, r=0.55 0.7) suggesting EBV titer, HOXA2 methylation and MMP9 level are inter-correlated with each other. Interestingly, the values of the three factors exhibit similar trends at a given time in a patient; however, the values vary at different time reflecting the clinical outcome or tumor burden of a patient during therapy. It appears that the higher the EBV copy number, the higher the HOXA2 methylation status and the higher the MMP-9 in the plasma of NPC patients. 


\section{DISCUSSION}

We herein identify two dysregulated transcription cascades that contribute to NPC tumorigenesis: (1) DNA methylation of HOXA2 impairs the binding of transcription activator $\mathrm{p} 300$ on the HOXA2 promoter; (2) the inactivation of $H O X A 2$ promotes TBP binding on $M M P-9$ promoter and activates the MMP-9 expression. Our data indicated that methylation of HOXA2 at the proximal p300-1 site interfered p300 binding, thereby suppressing HOXA2 expression. Although the core consensus sequence for $\mathrm{p} 300$ in the HOXA2 promoter (NNNG/AGGAGTNNNC/G) does not contain an obvious CG site; however, two CG sites were found flanking the central p300-1 sequence. Thus, methylation of flanking sequences could interfere with protein binding via steric hindrance.

The HOXA2, located within $H O X A$ gene cluster on human chromosome 7, is important for neural-crestderived structures development and the segmentation of neuromeres in hindbrain $[27,28]$. HOXA2 protein acts as a transcription regulator, and may suppress gene expression through the HOXA2-response element [13, 14]. Through direct binding to TAAT consensus sequence, HOX proteins may regulate the transcription of their target genes and thereby control cell differentiation during development [10,29]. Dysregulation of HOXA2 may have negative impacts on cells and/or lead to oncogenesis [30]. Aberrant expressions of $H O X$ genes have been correlated with breast cancer development and the malignant behavior of cancer cells [31]. Furthermore, the HOXA and HOXD clusters are hotspots for de novo methylation of $\mathrm{CpG}$ islands in human lung adenocarcinomas [32]. Temporal and spatial changes in HOX expression in developing and normal cells are tightly controlled. It would be interesting to test if other $H O X$ members are transcriptionally repressed by DNA methylation and/or functionally antagonized by TBP.

This is the first report to demonstrate HOXA2 can compete with the TBP for the TATA-box due to their DNA consensus sequence resemblance. Thereby, TBP and HOXA2 can co-regulate the $M M P-9$ transcription but they act in an opposite fashion. Conceivably, in addition to MMP-9, other HOXA2-TBP-targeted genes' transcription may also be affected in NPC. The MMP family has 29 members; among these members MMP-2 and MMP9 were found to have increased enzymatic activity and associations with metastasis in several cancers $[18,33]$ including NPC [21]. Two previous reports indicated that the MMP-9 overexpression observed in NPC was correlated with the EBV LMP1-mediated NF- $\mathrm{kB}$ signaling pathway [34] and the EBV Zta-mediated AP1 pathway [35]. Furthermore, increased MMP-9 expression in NPC tumors has been correlated with poor prognosis [36]. Our present findings offer an alternative explanation for MMP9 overexpression in NPC.
While normal healthy individuals have little cfDNA in their plasma $(\sim 30 \mathrm{ng} / \mathrm{ml})$, a high level of circulating cfDNA $(\sim 180 \mathrm{ng} / \mathrm{ml})$ can be detected in the plasma of cancer patients [26]. This difference reflects that cfDNA is mainly released from apoptotic and necrotic tumor cells; and the amount of cfDNA may be used as a cancer indicator [37]. As it is easier to obtain plasma samples than biopsies, measurement of aberrant methylated cfDNA in plasma may replace biopsies in NPC patients. Furthermore, the sensitivity and accuracy of MS-HRM is comparable to that of bisulfite sequencing suggesting that MS-HRM could be used as a rapid, sensitive and economical method for assessing HOXA2 methylation in both tumors and plasma samples of NPC patients. The 22 plasma samples collected from 5 NPC patients at different time intervals showed that EBV copy number, cfDNA HOXA2 methylation percentage and the level of MMP9 are inter-correlated. The dynamic levels of these three parameters vary over time within the same NPC patient and they may all serve as plasma biomarkers reflecting the tumor load of both in-situ and metastatic NPC tumors.

Finally, we propose a model in which illustrates aberrant hypermethylation of HOXA2 increases MMP-9 expression and promotes the invasiveness of NPC cells (Fig. 6). Silencing HOXA2 through DNA methylation may have profound effect on gene expression profile in NPC when considering there are $\sim 24 \%$ of the human genes which contain TATA-like element [38]. The final outcome for cells with HOXA2 impairment may therefore depend on the overall functions of all the downstream targets of HOXA2 and/or HOXA2-TBP.

\section{MATERIALS AND METHODS}

\section{Cell lines, NPC tissue samples and plasma}

The NPC cell lines, C666.1 [39] and HK1 [40], were provided by Dr. S. W. Tsao (Hong Kong University, SAR, China), and were cultured in $10 \%$ fetal bovine serum (FBS; Gibco)/RPMI1640 (Gibco BRL, Rockville, MD). The TW02 cell line was obtained from Dr. C. T. Lin (National Taiwan University, Taiwan); TW02 and the embryonic kidney cell line, 293T, were cultured in 10\% FBS/DMEM (Gibco). C666.1 and HK1 cells were treated with $10 \mu \mathrm{M}$ demethylation agent 5' Aza for 5 days, with daily replacement of fresh medium. Eight pairs of frozen NPC tumor and adjacent normal biopsies $(<2 \mathrm{~mm})$ were collected from Chang Gung Memorial Hospital (CGMH; Taiwan) by Dr. K. P. Chang. Genomic DNA and RNA of NPC biopsies were simultaneously extracted using the TRIZOL reagent (Invitrogen, Carlsbad, CA). Plasma was collected from five NPC patients at CGMH by Dr. C. L. Hsu. Plasma cell-free DNA was extracted by using QIAamp DNA kit (Qiagen, Germantown, MD). This 
study was reviewed and approved by the IRB and ethics committee of CGMH (IRB:97-1226A3 and IRB:950692B).

\section{Quantification of DNA methylation in NPC biopsies by MassArray MALDI-TOF MS analysis}

Genomic DNA $(2 \mu \mathrm{g})$ from all 16 tissue samples were bisulfite treated using the EZ DNA Methylation Kit (Zymo Research, Irvine, CA) and subjected to PCR amplification using three sets of HOXA2-specific bisulfite PCR primers. The extent of methylation at the individual $\mathrm{CpG}$ sites of these PCR products was further analyzed by Sequenom MassArray Epityper (Sequenom, San Diego CA) [41]. The degree of methylation for each $\mathrm{CpG}$ site was determined using the ratio between methylated- $\mathrm{C}$ and total cytosine, which ranged from 0 to 1 .

\section{Bisulfite sequencing}

Bisulfite-converted DNA (100 ng) was amplified using specific bisulfite sequencing (BS) primers that were designed using Methprimer (http://www.urogene.org/ methprimer/, UCSF). The resulting PCR products were cloned, and 8 10 individual clones were sequenced.

\section{Cell proliferation and invasion assay}

Cell proliferation was determined by trypan blue (Gibco) exclusion and cell counting. Stable HOXA2expressing and vector control HK1 $\left(2 \times 10^{5}\right)$ or TW04 $\left(5 \times 10^{4}\right)$ cells were seeded in 6-well plates, and cell numbers were counted daily for 5 days. Cell invasion was assessed using a cell invasion assay kit (Chemicon, Temecula, CA). Briefly, HOXA2-expressing HK1 (5x10 $)$ or TW02 cells $\left(4 \times 10^{5}\right)$ were seeded to the upper chamber and cultured for $24 \mathrm{hr}$ (TW02) or $72 \mathrm{hr}$ (HK1), allowing cells to migrate through the $0.8-\mu \mathrm{m}$ membrane. The membrane was then excised and stained with $0.1 \%$ crystal violet; and cells were counted under a microscope. All experiments were repeated at least three times, each in duplicate.

\section{Promoter analysis and luciferase assay}

The HOXA2 and MMP-9 luciferase reporters, pHOXA2/pGL3 and pMMP-9/pGL3, were amplified and both subcloned into the BglII and HindIII sites of the pGL3-basic vector (Promega, Madison, WI). To normalize each transfection reaction, a renilla reporter plasmid ( $\mathrm{pRL}$ CMV; Promega) (one-tenth of the total DNA amount) was co-transfected with the luciferase reporters. Cells were harvested $48 \mathrm{hr}$ post-transfection and lysed, and promoter activity was assayed using the Dual Luciferase Assay
System (Promega) on a GLOMAX 20/20 Luminometer (Promega).

\section{Antibodies for Western blot analyses}

Primary antibodies: anti-Flag antibody (M2; Sigma), p300 antibody (Santa Cruz Biotech, Santa Cruz, CA), and anti-actin monoclonal antibody (MDBio, Taipei, Taiwan) and HRP-conjugated secondary antibodies (goat antimouse antibody and goat anti-rabbit antibody) were used. The results were visualized using an ECL detection kit (Millipore, Billerica, MA).

\section{Quantitative chromatin immunoprecipitation (ChIP) assay}

ChIP assays were performed as previously described [42]. Either $1 \mu \mathrm{g}$ of anti-HOXA2 antibody or $2 \mu \mathrm{g}$ of the other specific antibodies (anti-p300, Santa Cruz; Flag® M2, Sigma; anti-TBP, Santa Cruz; anti-RNA polymerase II, Covance) or control IgG were used for immunoprecipatation. The DNA fragments were purified using a QIAquick PCR purification kit (Qiagen, Hilden, Germany), and Q-PCR was performed using primers against appropriate promoter regions. The quantitative ChIP signals (Ct values) were normalized with respect to the input control.

\section{Gelatin zymography assay}

Cells were seeded in 6-well plates, grown to confluence, washed three times with serum-free medium containing $0.1 \%$ bovine serum albumin (Sigma), and preincubated with serum-free medium for $3 \mathrm{hr}$. The culture medium was collected and diluted 1:1 (v/v) with 2x SDS sample buffer and heated for $30 \mathrm{~min}$ at $37^{\circ} \mathrm{C}$. The diluted medium $(50 \mu \mathrm{l})$ was separated on an $8 \%$ SDS-PAGE gel containing $1 \mathrm{mg} / \mathrm{ml}$ gelatin. The gel was washed twice with $2.5 \%$ Triton X-100, incubated for $15 \mathrm{~min}$ with developing buffer $(0.2 \mathrm{M} \mathrm{NaCl}, 0.04 \mathrm{M}$ Tris-HCl, and $\left.0.01 \mathrm{M} \mathrm{CaCl}_{2}, \mathrm{pH} 7.5\right)$ and transferred to fresh developing buffer for $48 \mathrm{hr}$. The gel was fixed in fixing buffer [ $45 \%$ (v/v) methanol, and 10\% (v/v) acetic acid], stained with $0.125 \%$ Coomassie blue, and destained for visualization of the gelatin-specific enzyme bands.

\section{DNA pull-down assay}

DNA pull-down assays were performed as previously described [42], with some modification. The double-stranded annealed biotinylated probes (100 250 pmole) were incubated with $25 \mu \mathrm{l}$ M-280 Streptavidin Dynabeads (Invitrogen) in recommended binding buffer for $1 \mathrm{hr}$ at room temperature. In parallel, 100 250 $\mu \mathrm{g}$ 
nuclear extracts and salmon sperm DNA $2.5 \mu \mathrm{g}$ were incubated with $25 \mu$ unconjugated Dynabeads (Invitrogen) for $30 \mathrm{~min}$ at room temperature. The pre-cleared nuclear extract was incubated with the probe-bounded beads for 1 $\mathrm{hr}$ at room temperature. The Dynabead-bound complexes were washed six times with binding buffer containing $0.5 \%$ NP-40, and the DNA-bound proteins were eluted in SDS sample buffer and assayed by Western blotting.

\section{MMP-9 ELISA}

Plasma samples collected from four NPC patients at different time points were diluted 40-fold with Calibrator Diluent buffer (R\&D Systems, Minneapolis $\mathrm{MN})$. Standards, controls and samples were added to microplate and incubated for $2 \mathrm{hr}$ at room temperature. The samples were then washed three times and incubated with the substrate solution. The amount of MMP-9 in the samples was measured by ELISA reader at O.D. $450 \mathrm{~nm}$, and compared with the standards.

\section{ACKNOWLEDGEMENTS}

We thank Dr. Li-Jung Juan for constructive criticism; Dr. Chin-Tarng Lin and Dr. Sai-Wah Tsao for providing NPC cell lines; Chun-Nan OuYang and Hsing$\mathrm{Ju}$ Lin for technical assistant.

\section{FUNDING}

This work was supported by grants from Chang Gung Memorial Hospital, Taiwan, CMRPD170371, CMRPD180391 and CMRPD1A0351; National Sciences Council of Republic of China (ROC), NSC982320B182033MY2 (NMRPD180981 \& NMRPD180982); and Ministry of Education (ROC), EMRPD1A0721, EMRPD1B0231, EMRPD1C0201.

\section{REFERENCES}

1. Wei WI and Sham JS. Nasopharyngeal carcinoma. Lancet. 2005; 365(9476):2041-2054.

2. Spano JP, Busson P, Atlan D, Bourhis J, Pignon JP, Esteban $\mathrm{C}$ and Armand JP. Nasopharyngeal carcinomas: an update. European journal of cancer. 2003; 39(15):2121-2135.

3. Tse KP, Su WH, Chang KP, Tsang NM, Yu CJ, Tang P, See LC, Hsueh C, Yang ML, Hao SP, Li HY, Wang MH, Liao LP, Chen LC, Lin SR, Jorgensen TJ, et al. Genomewide association study reveals multiple nasopharyngeal carcinoma-associated loci within the HLA region at chromosome 6p21.3. American journal of human genetics. 2009; 85(2):194-203.

4. Thorley-Lawson DA and Allday MJ. The curious case of the tumour virus: 50 years of Burkitt's lymphoma. Nature reviews Microbiology. 2008; 6(12):913-924.

5. Tsai CN, Tsai CL, Tse KP, Chang HY and Chang YS. The Epstein-Barr virus oncogene product, latent membrane protein 1, induces the downregulation of E-cadherin gene expression via activation of DNA methyltransferases. Proc Natl Acad Sci U S A. 2002; 99(15):10084-10089.

6. Tsai CL, Li HP, Lu YJ, Hsueh C, Liang Y, Chen CL, Tsao SW, Tse KP, Yu JS and Chang YS. Activation of DNA methyltransferase 1 by EBV LMP1 Involves c-Jun NH(2)terminal kinase signaling. Cancer Res. 2006; 66(24):1166811676.

7. Esteller M. Epigenetics in cancer. N Engl J Med. 2008; 358(11):1148-1159.

8. Lopez-Serra L and Esteller M. Proteins that bind methylated DNA and human cancer: reading the wrong words. British journal of cancer. 2008; 98(12):1881-1885.

9. Jones PA. Functions of DNA methylation: islands, start sites, gene bodies and beyond. Nature reviews Genetics. 2012; 13(7):484-492.

10. Akin $\mathrm{ZN}$ and Nazarali AJ. Hox genes and their candidate downstream targets in the developing central nervous system. Cellular and molecular neurobiology. 2005; 25(34):697-741.

11. Lappin TR, Grier DG, Thompson A and Halliday HL. HOX genes: seductive science, mysterious mechanisms. Ulster Med J. 2006; 75(1):23-31.

12. Grier DG, Thompson A, Kwasniewska A, McGonigle GJ, Halliday HL and Lappin TR. The pathophysiology of HOX genes and their role in cancer. The Journal of pathology. 2005; 205(2):154-171.

13. Kutejova E, Engist B, Self M, Oliver G, Kirilenko P and Bobola N. Six2 functions redundantly immediately downstream of Hoxa2. Development. 2008; 135(8):14631470 .

14. Lampe X, Picard JJ and Rezsohazy R. The Hoxa2 enhancer 2 contains a critical Hoxa2 responsive regulatory element. Biochem Biophys Res Commun. 2004; 316(3):898-902.

15. Li LC and Dahiya R. MethPrimer: designing primers for methylation PCRs. Bioinformatics. 2002; 18(11):14271431.

16. Hsieh CL. Stability of patch methylation and its impact in regions of transcriptional initiation and elongation. Molecular and cellular biology. 1997; 17(10):5897-5904.

17. Chan HM and La Thangue NB. p300/CBP proteins: HATs for transcriptional bridges and scaffolds. Journal of cell science. 2001; 114(Pt 13):2363-2373.

18. Nelson AR, Fingleton B, Rothenberg ML and Matrisian LM. Matrix metalloproteinases: biologic activity and clinical implications. J Clin Oncol. 2000; 18(5):1135-1149.

19. Tang JG, Li $X$ and Chen P. Expression of matrix metalloproteinase 9 in nasopharyngeal carcinoma and association with Epstein-Barr virus infection. J Zhejiang Univ Sci. 2004; 5(10):1304-1312.

20. Takeshita H, Yoshizaki T, Miller WE, Sato H, Furukawa 
M, Pagano JS and Raab-Traub N. Matrix metalloproteinase 9 expression is induced by Epstein-Barr virus latent membrane protein $1 \mathrm{C}$-terminal activation regions 1 and 2 . J Virol. 1999; 73(7):5548-5555.

21. Lee DC, Chua DT, Wei WI, Sham JS and Lau AS. Induction of matrix metalloproteinases by Epstein-Barr virus latent membrane protein 1 isolated from nasopharyngeal carcinoma. Biomed Pharmacother. 2007; 61(9):520-526.

22. Smith TM, Wang X, Zhang W, Kulyk W and Nazarali AJ. Hoxa2 plays a direct role in murine palate development. Dev Dyn. 2009; 238(9):2364-2373.

23. Yao J, Xiong S, Klos K, Nguyen N, Grijalva R, Li P and $\mathrm{Yu}$ D. Multiple signaling pathways involved in activation of matrix metalloproteinase-9 (MMP-9) by heregulin-beta1 in human breast cancer cells. Oncogene. 2001; 20(56):80668074.

24. Kirilenko P, He G, Mankoo BS, Mallo M, Jones R and Bobola N. Transient activation of meox 1 is an early component of the gene regulatory network downstream of hoxa2. Molecular and cellular biology. 2011; 31(6):13011308.

25. Kutejova E, Engist B, Mallo M, Kanzler B and Bobola N. Hoxa2 downregulates Six2 in the neural crest-derived mesenchyme. Development. 2005; 132(3):469-478.

26. Schwarzenbach $H$, Chun FK, Isbarn H, Huland $H$ and Pantel K. Genomic profiling of cell-free DNA in blood and bone marrow of prostate cancer patients. Journal of cancer research and clinical oncology. 2011; 137(5):811-819.

27. Davenne M, Maconochie MK, Neun R, Pattyn A, Chambon P, Krumlauf R and Rijli FM. Hoxa2 and Hoxb2 control dorsoventral patterns of neuronal development in the rostral hindbrain. Neuron. 1999; 22(4):677-691.

28. Ohnemus S, Bobola N, Kanzler B and Mallo M. Different levels of Hoxa2 are required for particular developmental processes. Mechanisms of development. 2001; 108(12):135-147.

29. Zappavigna V, Sartori D and Mavilio F. Specificity of HOX protein function depends on DNA-protein and proteinprotein interactions, both mediated by the homeo domain. Genes \& development. 1994; 8(6):732-744.

30. Cillo C, Faiella A, Cantile M and Boncinelli E. Homeobox genes and cancer. Exp Cell Res. 1999; 248(1):1-9.

31. Makiyama K, Hamada J, Takada M, Murakawa K, Takahashi Y, Tada M, Tamoto E, Shindo G, Matsunaga A, Teramoto K, Komuro K, Kondo S, Katoh H, Koike T and Moriuchi T. Aberrant expression of HOX genes in human invasive breast carcinoma. Oncol Rep. 2005; 13(4):673679.

32. Shiraishi M, Sekiguchi A, Oates AJ, Terry MJ and Miyamoto Y. HOX gene clusters are hotspots of de novo methylation in $\mathrm{CpG}$ islands of human lung adenocarcinomas. Oncogene. 2002; 21(22):3659-3662.

33. Imanishi Y, Fujii M, Tokumaru Y, Tomita T, Kanke M, Kanzaki J, Kameyama K, Otani Y and Sato H. Clinical significance of expression of membrane type 1 matrix metalloproteinase and matrix metalloproteinase- 2 in human head and neck squamous cell carcinoma. Hum Pathol. 2000; 31(8):895-904.

34. Yoshizaki T, Sato H, Furukawa M and Pagano JS. The expression of matrix metalloproteinase 9 is enhanced by Epstein-Barr virus latent membrane protein 1. Proceedings of the National Academy of Sciences of the United States of America. 1998; 95(7):3621-3626.

35. Yoshizaki T, Sato H, Murono S, Pagano JS and Furukawa M. Matrix metalloproteinase 9 is induced by the EpsteinBarr virus BZLF1 transactivator. Clinical \& experimental metastasis. 1999; 17(5):431-436.

36. Liu Z, Li L, Yang Z, Luo W, Li X, Yang H, Yao K, Wu B and Fang W. Increased expression of MMP9 is correlated with poor prognosis of nasopharyngeal carcinoma. BMC Cancer. 2010; 10:270.

37. Hsu CL, Chang KP, Lin CY, Chang HK, Wang CH, Lin TL, Liao CT, Tsang NM, Lee LY, Chan SC, Ng SH, Li HP, Chang YS and Wang HM. Plasma Epstein-Barr virus DNA concentration and clearance rate as novel prognostic factors for metastatic nasopharyngeal carcinoma. Head \& neck. 2012; 34(8):1064-1070.

38. Yang C, Bolotin E, Jiang T, Sladek FM and Martinez E. Prevalence of the initiator over the TATA box in human and yeast genes and identification of DNA motifs enriched in human TATA-less core promoters. Gene. 2007; 389(1):5265.

39. Cheung ST, Huang DP, Hui AB, Lo KW, Ko CW, Tsang YS, Wong N, Whitney BM and Lee JC. Nasopharyngeal carcinoma cell line (C666-1) consistently harbouring Epstein-Barr virus. Int J Cancer. 1999; 83(1):121-126.

40. Huang DP, Ho JH, Poon YF, Chew EC, Saw D, Lui M, Li CL, Mak LS, Lai SH and Lau WH. Establishment of a cell line (NPC/HK1) from a differentiated squamous carcinoma of the nasopharynx. Int J Cancer. 1980; 26(2):127-132.

41. Ehrich M, Turner J, Gibbs P, Lipton L, Giovanneti M, Cantor $\mathrm{C}$ and van den Boom D. Cytosine methylation profiling of cancer cell lines. Proceedings of the National Academy of Sciences of the United States of America. 2008; 105(12):4844-4849.

42. Chen LC, Chung IC, Hsueh C, Tsang NM, Chi LM, Liang Y, Chen CC, Wang LJ and Chang YS. The antiapoptotic protein, FLIP, is regulated by heterogeneous nuclear ribonucleoprotein $\mathrm{K}$ and correlates with poor overall survival of nasopharyngeal carcinoma patients. Cell death and differentiation. 2010; 17(9):1463-1473. 